\title{
Computer models for fuel element behaviour
}

\author{
J R MATTHEWS \\ Theoretical Physics Division, AERE Harwell, UK
}

\begin{abstract}
Over the past 20 years computer modelling of fuel performance has developed into a well-established procedure, which has been valuable in the understanding and the improvement of fuel rod behaviour. The range of computer models presently applied to cylindrical ceramic fuels is reviewed. A critical appraisal is made of the numerical techniques used for mechanical and thermal analysis. The necessity for benchmark calculations is emphasized and various approaches for model validation are discussed. A number of special topics are chosen for deeper discussion. These include: improved description of cladding deformation and the estimation of failure; the analysis of stress concentrations in the cladding; fission gas analysis; and chemical modelling. Finally some speculation is made on the future of fuel rod modelling.
\end{abstract}

Keywords. Computer modelling; nuclear fuels; oxide fuels; deformation; failure; heat transfer; fission products; fission gases.

\section{Introduction}

This paper will give a very personal view of the subject of fuel element computer modelling. It will look critically at the development of computer programs for the simulation of the behaviour of cylindrical fuel rods containing ceramic fuel under normal and accident conditions. On this basis particulate fuels, metal fuels and cermet fuels will not be considered. To limit the scope somewhat, the behaviour of individual fuel rods will be discussed and the subject of rod-to-rod interactions or fuel rod interactions with other structural components will not be covered.

The development of computer models for fuel performance has its origins in the mid1960 s when, at a number of centres, heat transfer and fuel rod mechanics calculations were coupled for the first time. The best known of these early models is CYGRo, which laid out the structure that future models followed (Freidrich 1965). During the next five years there was an explosion of activity when almost every research centre in the USA, Japan and Western Europe produced its own fuel performance model; for a list of the models of this period see Matthews (1972). Some of the names of the computer codes written at that time are still used, such as SATURN, COMETHE, LIFE and CYGRO, but the methods used within them have in general been greatly improved.

During the 1970s there was a growing interest in reactor safety and many of the codes were extended for use in accident calculations. There was also an interest in producing more detailed codes that permitted the study of microstructural processes within the fuel and its cladding. More recently the decline of nuclear power plant ordering in the USA has limited the number of active centres in that country. Interest has remained strong in Western Europe and Japan and there have also been encouraging developments in Eastern Europe, Latin America and India. For recent reviews of water reactor fuel element modelling for performance and safety studies see Gittus (1982, 1984). 
The content of fuel behaviour codes varies enormously and they range from very simple empirical models with the minimum of computational framework to complex detailed calculations including many physical and chemical sub-models. The simpler codes tend to be aimed at the fuel of a single reactor type, or even a single fuel variant within that type. The more physically based codes are often applied to a wide range of fuels. This is not surprising as most power reactors use fuel of similar configuration: i.e. a cylindrical metal cladding tube filled with cylindrical $\mathrm{UO}_{2}$ or $(\mathrm{U}, \mathrm{Pu}) \mathrm{O}_{2}$ pellets. This is the case for fast breeder reactors, the Ux advanced gas cooled reactor, the pressurised water reactor, the boiling water reactor and the various heavy water reactors.

\section{Range of fuel behaviour codes}

It would be impossible in this short paper to list all the current fuel codes and not much would be gained without a reasonably detailed assessment of each. However it is worth looking at the range of models and classifying them with a few selected examples. Most models attempt to represent a fuel rod by assembling a series of transverse slices or zones and connecting them loosely by pin pressure, axial loading conditions and the coolant heat transfer (see figure 1). This is often referred to as the $1 \frac{1}{2}$-dimensional approximation. A number of codes use two-dimensional finite-element analyses to investigate specific aspects of behaviour such as clad interaction with pellet "hourglassing" or local stress concentrations near cracks, e.g. SAFENR (Chhatre et al 1983) or MIPAC(Ichikawa et al 1980). The modelling of whole pin behaviour however is wasteful because the local stress concentrations occur on a scale that is too small to be usefully covered. Often such calculations are done by coupling with a $1 \frac{1}{2}$-dimensional code.

The $1 \frac{1}{2}$-dimensional codes fall into a number of types. There are simple performance codes that do not have complicated mechanics models, but concentrate on temperature calculation and the development of fuel restructuring and fission gas release, e.g. the us fast reactor code sIEx (Dutt and Baker 1975) and the UX thermal reactor code MINIPAT (Hughes et al 1982). Such simple codes are also often used to supply the starting conditions for accident analysis codes, i.e. FRAPCON (Berna et al 1980). Then there are detailed performance codes that include descriptions of the deformation of both the fuel and cladding by creep and plastic flow, as well as thermal and restructuring calculations, e.g. the UK code FRUMP (Wilmore and Matthews 1979), the Argentinian code BACo (Harriague et al 1980) and the Indian code PROFESs (Sah and Venkatesh 1984). These codes also have some limited transient capability. The most complex are extremely versatile, e.g. URanus(Preusser and Lassmann 1983) which can be applied to oxide or carbide fuel, water or fast breeder reactors, transient or steady state, and in deterministic or probabilistic modes. Similarly the US LIFE-4 code (Stephen et al 1977) has been issued in transient, water reactor and carbide versions in addition to the fast breeder performance form it was originally written in. The COMETHE code (van Vliet and Meulemeester 1980) should also be mentioned as it has been widely used for analysis of performance and mild transients on water reactor and fast breeder fuel both in its country of origin and by EPRI in the USA.

In going to the field of safety analysis the range of codes is bridged by the TRAFIC code (Matthews 1984), which has the complete range fuel performance models, but also special modules for analysis of accident conditions. These include models for sodium boiling, molten fuel motion within the fuel pin, fuel ejection from the pin and a 


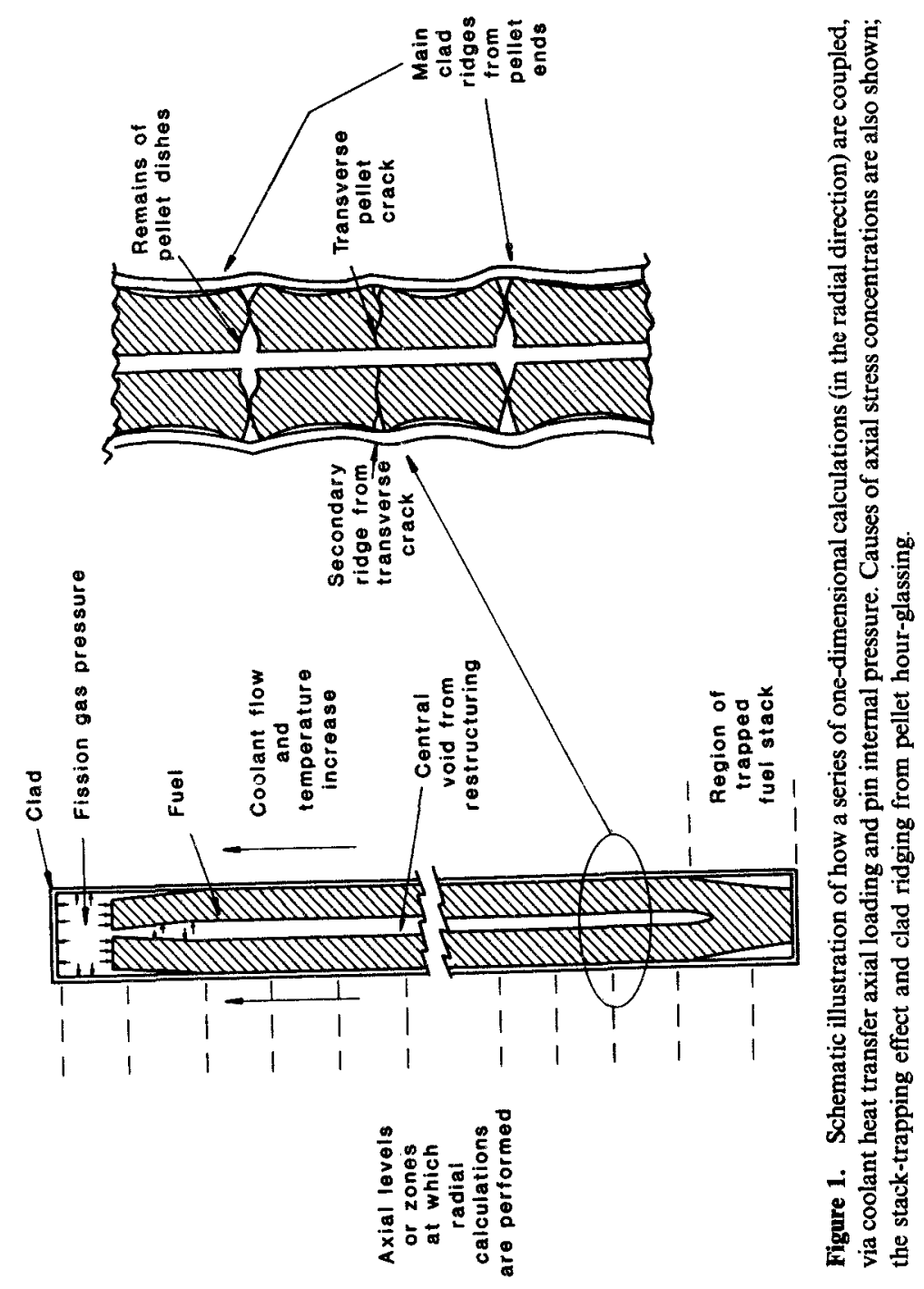


transient fission gas model (Matthews et al 1985). More specific accident models concentrate on the aspects of behaviour which are inherent in the type of fuel being studied. The fuel pin modelling within these codes is often simplified, as the main objective of the calculation is the blockage of coolant channels, the thermo-hydraulic problem and changes in the fuel rod configuration. For water reactors the most comprehensive code is FRAP-T6 (Seifken 1983). On the fast breeder reactor side the French Physura code (Melis 1982) and the Us code SAS-4A (Wider et al 1982) probably have the widest range of models. Most of the fast reactor models have recently been compared on a common test problem (Cacciabue et al 1982).

In addition to the whole pin models there are separate models that deal with a specific aspect of fuel rod behaviour. Many of these models can be coupled to whole pin models, although they are uneconomic for routine use. One example is fission gas behaviour analysis with codes such as GRASS and FASTGRASs (Rest 1979). Another is the detailed deformation analysis of Zircaloy and 316 stainless steel using the MATMOD programs (Schmidt and Miller 1981; Miller and Sherby 1977).

\section{The philosophy of fuel behaviour modelling}

Fuel rod behaviour modelling is one of the first examples of a complex physical system being simulated on the computer. For this reason it is worth examining the philosophy of modelling laying down some general principles to guide more effective future work.

First of all let us consider why such computer codes need to be constructed, rather than simpler calculations on specific aspects of behaviour such as temperature, fission gas release or cladding deformation. The reason is one of self-consistency. All aspects of the fuel behaviour are intimately connected. Take the example of fuel temperature. Fuel temperature is very sensitive to the size and condition of the fuel-cladding interface. Changes in the fuel temperature cause changes in the fuel size from thermal expansion and hence the size of the fuel-cladding gap and its heat transfer coefficient. Similarly release of fission gas from the fuel, which is sensitive to fuel temperature, alters the purity of the rod-filling gas, which changes the gas heat transfer coefficient and hence the fuel temperatures. Such interconnections make some sort of comprehensive fuel-rod model essential to the understanding of its behaviour. On a wider front whole pin models are also required to study the interactions with the coolant thermal and hydraulic behaviour. For the fast breeder reactor this is extended for safety analyses to calculation of the interactions with the reactivity of the reactor core.

The applications of fuel behaviour computer models are varied and it is essential to construct a model with appropriate characteristics. The following guidelines are worth adopting.

(i) The accuracy of the calculation should be sufficient. Methods should be chosen to give adequate accuracy, but the use of error control which is more stricter than necessary is inefficient.

(ii) The code should use sub-models which have consistent levels of approximation. It is no use coupling a sophisticated fission gas model or detailed finite-element model to a code which is basically incompatible.

(iii) No short-cuts should be taken on stability. The adding of extra models without proper coupling to avoid instabilities has led to problems in many codes. 
(iv) The code should be constructed in modular form to make the addition of submodels easy. This is not a trivial problem when stability is to be maintained.

(v) The main methods used in the code and all the sub-models should be verified (benchmarked) against standard or closed-form solutions. There has been at least one case of a major fuel-model being found to be at fault after several years of application.

(vi) All the controlling processes should be identified and included in the code. This is not so easy and is the key to effective modelling.

(vii) Computer codes should be identified by their materials properties, data-sets as well as their sub-models. Confusion often occurs because the data-set used was not clearly identified.

(viii) If empirical correlations are used as sub-models care should be taken that the correct assumptions were used when making them. This is an easily forgotten problem, e.g. when correlating gap conductance against fuel relocation the same models for fuel expansion and cracking should be used in making the correlations as in the code in which it is to be used; otherwise the effect could be included twice.

When constructing models, a decision has to be made on the degree of mechanistic detail which should be included in the code. Some codes are phenomenological but the processes identified are described by empirical correlations of the macroscopic processes. In this case the code itself can be calibrated by means of free parameters in the models. In practice this approach has not been very successful. The models can give a good description of the fuel-rod behaviour within the range of the data-base, but outside the deviations can be unsatisfactory. Such codes add little to the understanding of the system. A more satisfactory approach is to restrict empirical correlation to processes that can be evaluated by separate experiment. If the code then gives bad predictions it means that some controlling process or interaction has been omitted. Fuel behaviour codes for design purposes or for use as part of a larger thermohydraulics or accident code, do not need detailed physically based sub-models. Codes used for experiment interpretation should be based on sub-models with a sound physical basis. Detailed modelling is very valuable in determining. what the controlling processes are, and ensuring that simpler models have identified ranges of application.

\section{Methods for thermal and mechanical calculations}

The number of numerical methods for solving the basic heat transfer and fuel rod mechanics problems is almost as many as there are fuel codes. These, however, break down into three basic techniques on closer examination. Numerical approximations are required because most of the properties controlling behaviour are sensitive to temperature and to other physical variables which are functions of position. The three techniques are: (i) the finite difference approximation, where the differential equations for the system are approximated by difference expansions; (ii) the finite element approximation, where trial functions representing the variables being calculated are made to fit the differential equations of the system approximately over limited regions and then the whole system is made self-consistent; and (iii) the use of analytical or semianalytical solutions to represent the behaviour over rings of the material which are then made self-consistent over the whole system. There are a host of variations on these 
methods by taking different orders of difference approximation, integration techniques, orders of trial function and the choice of variables to represent the system. Currently there is a move towards the use of finite element methods because of the ease with which the variation in properties over the system can be accommodated. Finite element methods tend to be simpler to formulate, but care has to be taken when interpolating values from the solutions as the values taken from the trial functions can sometimes be misleading at positions other than integration points

The heat transfer problem within the fuel pin presents few problems, even taking account of transients, apart from where melting takes place. Care has to be taken to ensure that enthalpy is conserved when fixing the position of the melting front (Matthews 1979).

The description of the mechanical behaviour of the fuel rod is a much more difficult problem. One of the main difficulties arises from the very large variation in creep properties in the fuel, because of the sensitivity of creep to temperature. The creep strength of the fuel can vary typically by 10 orders of magnitude in a highly rated fuel design, in going from the cooler outer fuel regions dominated by irradiation creep to the hot plastic centre. This caused many problems in early models. One standard solution to the problem was to partition the fuel, above a somewhat arbitrary temperature, into a plastic zone where all shear stresses are relaxed. Despite this, the time-steps taken by the codes were often unacceptably small. One method of overcoming this problem was to extrapolate the total strains calculated by the codes to the next time-step and then solve for the stresses at the end of the time-step to enable a better estimate of the creep strain to be obtained (Jankus and Weeks 1970). This method works well for many cases and especially where the strain increment is controlled by a change in temperature or a swelling increment which is a weak function of the stress in the system. The method, however, breaks down in some circumstances and particularly for the case of the creep of the cladding tube by fission gas pressure (Nimo and Ogawra 1980). The only sound solution is to use a method which has a strong implicit component and is guaranteed stable. The Crank-Nicholson or Euler backward approximations are adequate for most applications, but the problem of dealing with nonlinearities in the response of the system has to be faced.

The simplest method of linearising the problem is to use the Newton-Raphson approximation. Many workers have avoided implicit solutions and the use of the Newton-Raphson method because they interfered with the modular nature of the code and were complicated to implement. Recent work has shown that this need not be the case (Matthews 1983) and the TRAFIC code is fully modular and uses a fully implicit method of solution. One aspect to be careful of is the treatment of fuel hot-pressing and stress-dependent fuel swelling. Some codes take care to deal with the problem of fuel creep, but then find there are stability problems when a new fuel densification or swelling model is added to the code. The same criteria should be applied to the calculation of fuel volume changes as those used to implement creep deformation.

There are many other problems associated with the fuel-rods mechanics calculation, and it is worth quickly listing a few. Fuel strains are often large, even if cladding strains are small, and care must be taken to make the deformation account for finite strains. In some cases the plastic strains in the fuel or clad are very large compared with the level of elastic strain in the system. Some finite element techniques become inaccurate in this situation and an additional degree of freedom should be introduced into the solution to avoid this (Hermmann 1965). Fuel cracking is an important factor in determining the 
fuel-cladding gap and reducing the fuel-cladding mechanical interaction (FCMI). Some models calculate no FCMI prior to the closure of the cracks. This will underestimate the cladding strains and as will be seen below the cracks play an important role in fuel-rod failure. Some codes neglect transverse cracks, lying normal to the axial direction; in doing so they will underestimate fuel relocation and miss important effects on fuel stack axial-loading (see figure 1).

Finally the interaction between the thermal and mechanical calculations must not be forgotien. In most codes the thermal calculation is performed first for a time-step followed by the mechanics, with iterations if necessary. This can result, when the fuelcladding gap is small, in an oscillating instability. This problem is only encountered occasionally and can be countered by introducing some form of numerical damping into the calculation. A more rigorous approach would be to couple the thermal and mechanical calculations; a possibility that we will return to in the discussion on future developments.

\section{Special topics}

In this section we discuss recent developments and requirements in a few selected areas of interest.

\subsection{Improved models for cladding deformation and failure}

The deformation and rupture of fuel-rod cladding presents perhaps the greatest challenge to fuel behaviour modelling. Up to now cladding deformation has been included in codes by simple correlations on creep and plastic deformation, supplemented by corrections for radiation hardening and empirical representation of irradiation creep, the growth of Zircaloy or void swelling of steels in fast breeder reactors. In fact the dimensional changes that a metal alloy will undergo under an applied stress and a simultaneous exposure to fast neutron damage are extremely complex. Figure 2 illustrates this. Some of the effects have been recently reviewed by Bullough (1984). Irradiation changes the dislocation structure, alters the distribution of both the major and minor constituents of the alloy and produces a range of embrittling effects. Added to this is the effect of the presence of fission products adjacent to the cladding inner surface.

To make sense of these interactions new techniques are required. Simple semiempirical models can be constructed, which are based on the actual physical processes taking place. In this way several processes like irradiation creep, dislocation structure evolution, growth, void swelling and thermal creep can all be interlinked (Bullough et al 1981). The model can then be used as the basis of an empirical correlation, but with the minimum of free variables. This should increase the ability of modelling to reproduce the observed behaviour of fuel-rods and remove some of the present discrepancies.

\subsection{Stress concentration and failure}

Failure of thermal reactor fuel rods is often dominated by local stress concentrations. There are two main sources; the ends of pellets (see figure 1) and the regions of cladding 

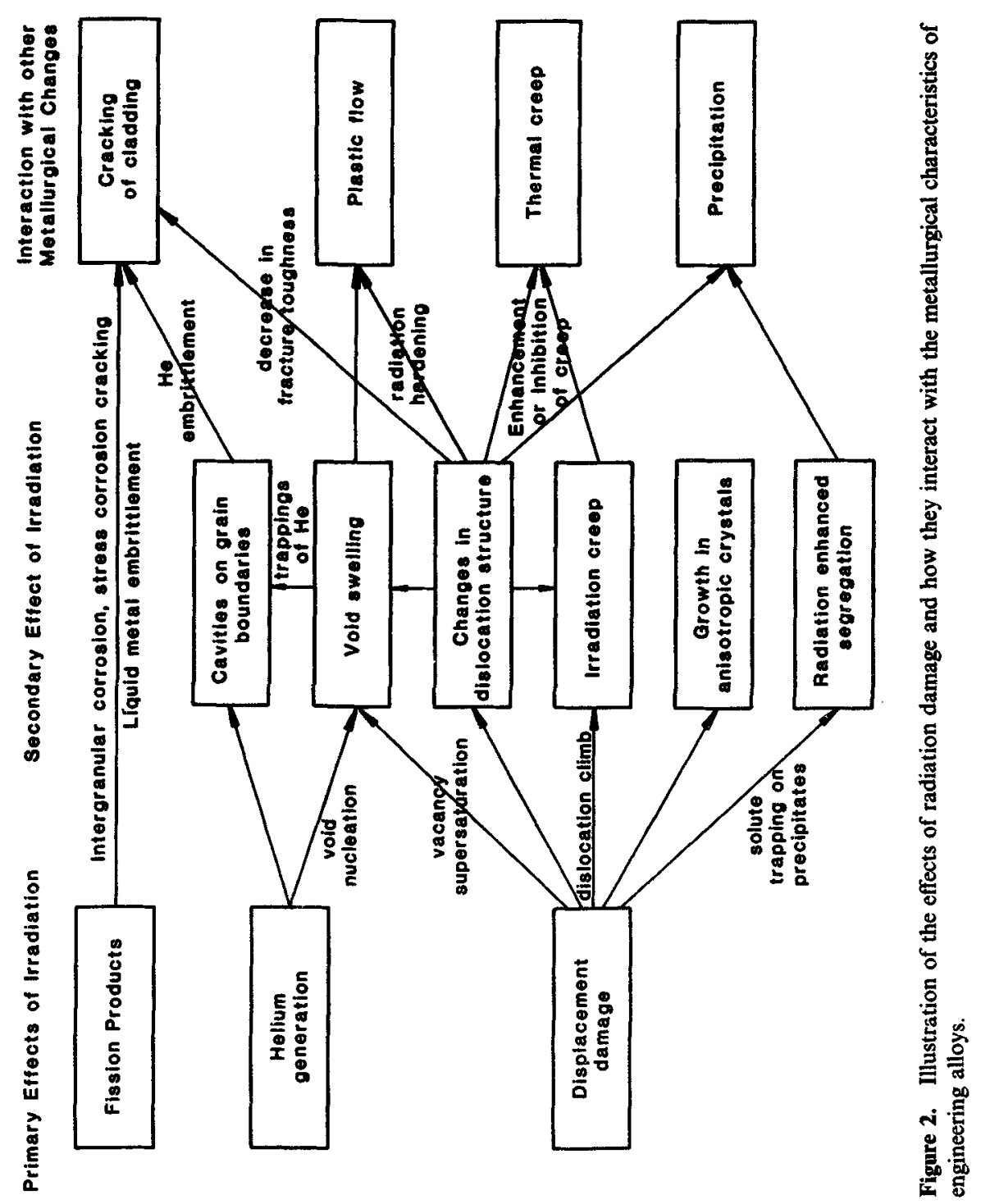
adjacent to radial cracks in fuel pellets (see figure 3 ). The most widely used model for stress concentrations over cracks is that of Gittus (Gittus et al 1970; Gittus 1972). Although found useful in practice this model has several deficiencies. The stress equilibrium conditions in the cladding shell are not soundly based. To do this properly is difficult and complicated and much of the elegance of Gittus' solutions is lost (Tromans and Matthews 1984). The treatment neglects bending moments in the cladding, which are often dominant (Nakatsuka 1981). The solutions to the friction problem should have a stuck region and a sliding region, in order to satisfy cyclic boundary conditions (figure 3 ). The stress concentration extends only over the region of the cladding over the crack and where sliding takes place (figure 4). The extent of the sliding region is sensitive to the external coolant pressure. The size of the stress concentration on the cladding inner surface is greatly underestimated by the thin shell model, as the through wall stress gradients are large. Only rigorous two-dimensional calculations are able to fix the inner stress accurately. The stress concentration is over-

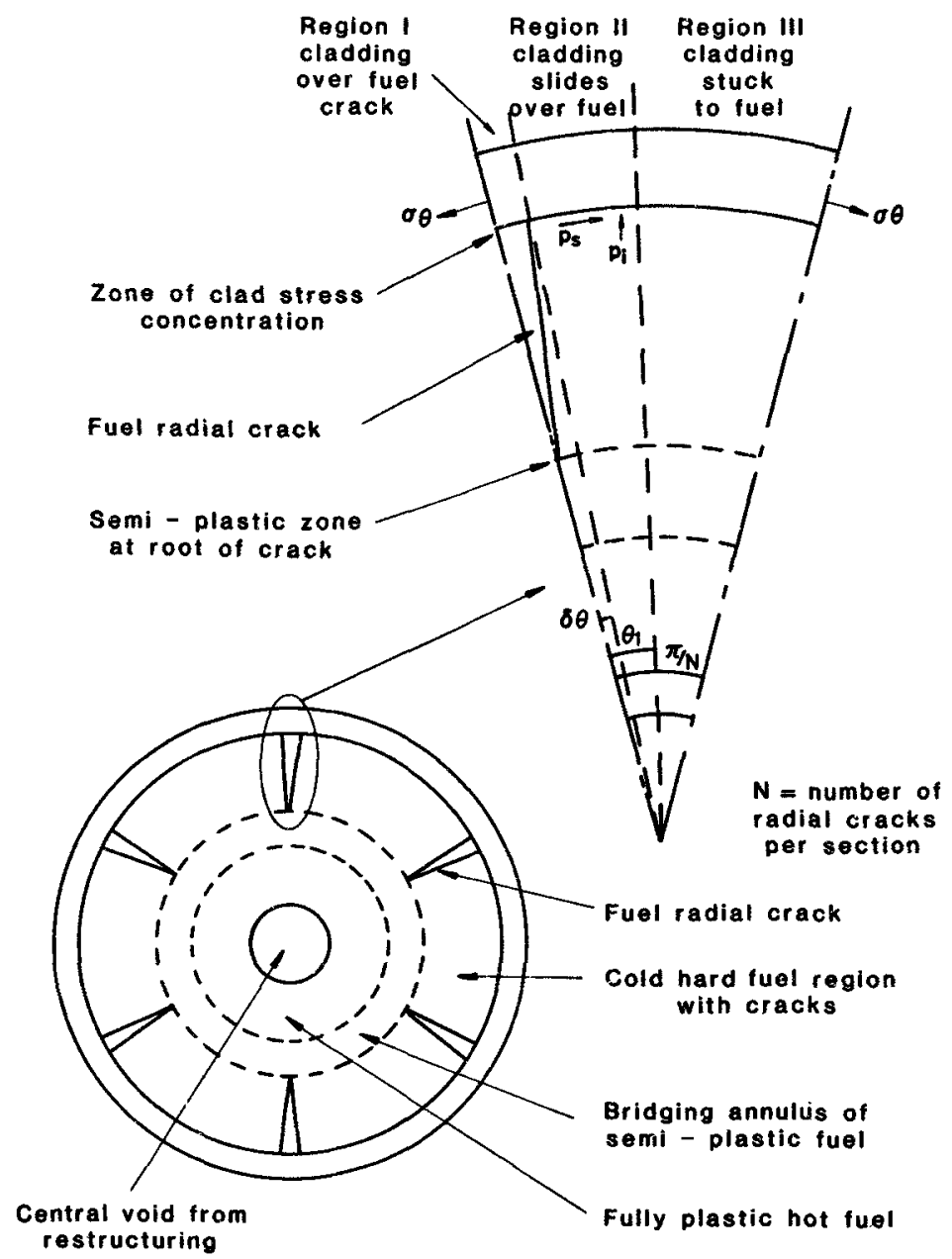

Figure 3. Schematic illustration of the relationship between fuel radial cracking, fuel structural zones and stress concentrations in cladding. 


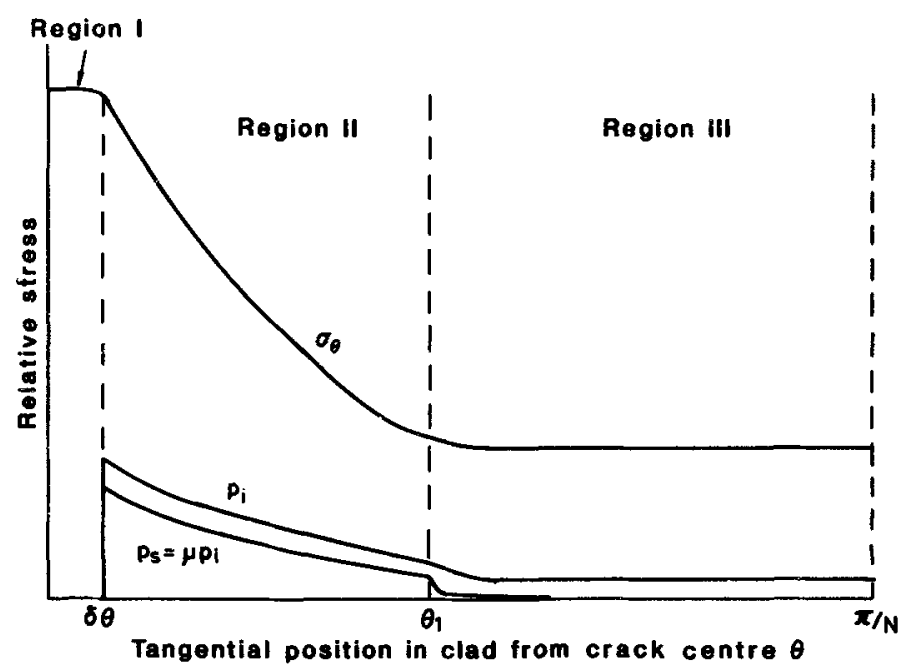

Figure 4. Schematic illustration of clad mean hoop stress, normal pressure and shear traction, vary with angular position, when sliding and sticking occur between fuel and cladding.

estimated, however, because the reaction of the fuel is neglected. Even if the fuel adjacent to the cladding is hard, the fuel at the crack roots may be plastic and reduce the load on the cladding (Stephen et al 1979). The stress concentration at the cladding does not arise from the radial displacement of the fuel, but from the relative opening of the cracks, which occurs from thermal expansion on power increases and from differential swelling (Tromans and Matthews 1984). Finally the simple shell model does not include other sources of internal stress in the cladding, such as thermal stresses, which will be important when nonlinear deformation is taking place.

Many of these remarks also apply to simple analyses of cladding interactions with the pellet ends. The lesson is that this is an example where simple one-dimensional calculations are inadequate. Guidelines for the effect of clad-ridging from pellet interactions or stress concentrations over cracks, should be carried out with the appropriate two-dimensional finite-element model of the fuel and clad together.

These aspects of local cladding stress concentrations are not so important in fast reactor fuel because: the coolant pressure is low; the cladding is relatively thick and strong; the fuel is more highly rated and softer with many more radial and transverse cracks; and stress corrosion cracking has not yet been recognized as a problem. The main problem with strain concentrations in fast reactor cladding arises from local temperature perturbations or hot-spots. These occur because of the high surface heat fluxes on fast reactor fuel-rods and because of the problem with fast neutron induced swelling of the cladding causing distortions of the fuel bundle.

\subsection{Fission gas behaviour}

Fission gas swelling and release are important processes in determining fuel pin behaviour both under normal and accident conditions. Swelling is an important factor in FCMI and can also cushion rapid clad loading in overpower transient conditions. 
Fission gas release is also important as many fuel designs have limited plenum volumes to accommodate fission gases. Fast reactor fuel normally releases a high proportion of its fission gas inventory during normal high rating operation and many aspects of fuel behaviour in accidents are controlled by the retained gas in the fuel after melting (Wood et al 1984).

The processes involved in a complete description of fission gas behaviour are many and their interactions are complex (see figure 5). A recent review describes how these processes are handled in the OGRES and NEFIG models which form part of the TRAFIC code (Matthews and Wood 1984). There are, however, some indications that there may be some deficiencies in our understanding of fission gas behaviour in transients. Experiments both in the laboratory (Zimmermann 1984) and in-pile (Sontheimer et al 1984) give higher gas releases than anticipated. This was also evident in the DCOM blind problem that was reviewed at the Bowness-on-Windermere Conference in 1984. Current mechanistic fission gas models predict that on mild temperature transients most of the gas would be trapped in small bubbles and fission gas release would be small. There are several rival explanations of this discrepancy: the bubbles may become over-stuffed with gas and reject gas atoms, leaving a higher concentration in solution (Brearley et al 1984); grain boundary sweeping may be more important than previously thought (van Vliet and Billaux 1984); or the amount of gas held on the grain boundaries may be higher than previously thought. All of these explanations have their deficiencies. The observation of the growth of a coarse bubble population after high temperature annealing, suggests that what were previously thought to be small bubbles were in fact vacancy clusters which disappear before they trap gas (Matthews et al 1985). Hopefully these questions will be resolved in the next few years, as several experimental programmes are being conducted at present to provide more microstructural information.

\subsection{Chemical modelling}

Perhaps the most neglected aspect of fuel behaviour modelling has been fuel chemistry. Models so far have at most simple descriptions of oxygen redistribution within the fuel or simple calculations of caesium and plutonium migration. Chemical effects are very important in determining fuel-rod failure and better estimates of the fuel-cladding gap inventory of fission products and their chemical state are required. This is not a trivial problem as the number of chemical species and compounds involved is large. Among the problems are: calculations of the amounts of free iodine and tellurium as a function of linear rating and burn-up; good definition of the oxygen potential to the fuelcladding interface, which is particularly important for stainless steel clad rods; definition of the location and chemical state of caesium throughout the fuel rod; and good description of oxygen and plutonium redistribution during irradiation.

\section{Future developments}

This paper has looked at a range of important topics in the subject of fuel behaviour modelling, but there are insufficient space to discuss many other aspects that are just as important. For example we have neglected to discuss fuel restructuring processes and the behaviour of fuel rods during extreme accident conditions. To finish the paper it is worth briefly commenting on possible future trends. 


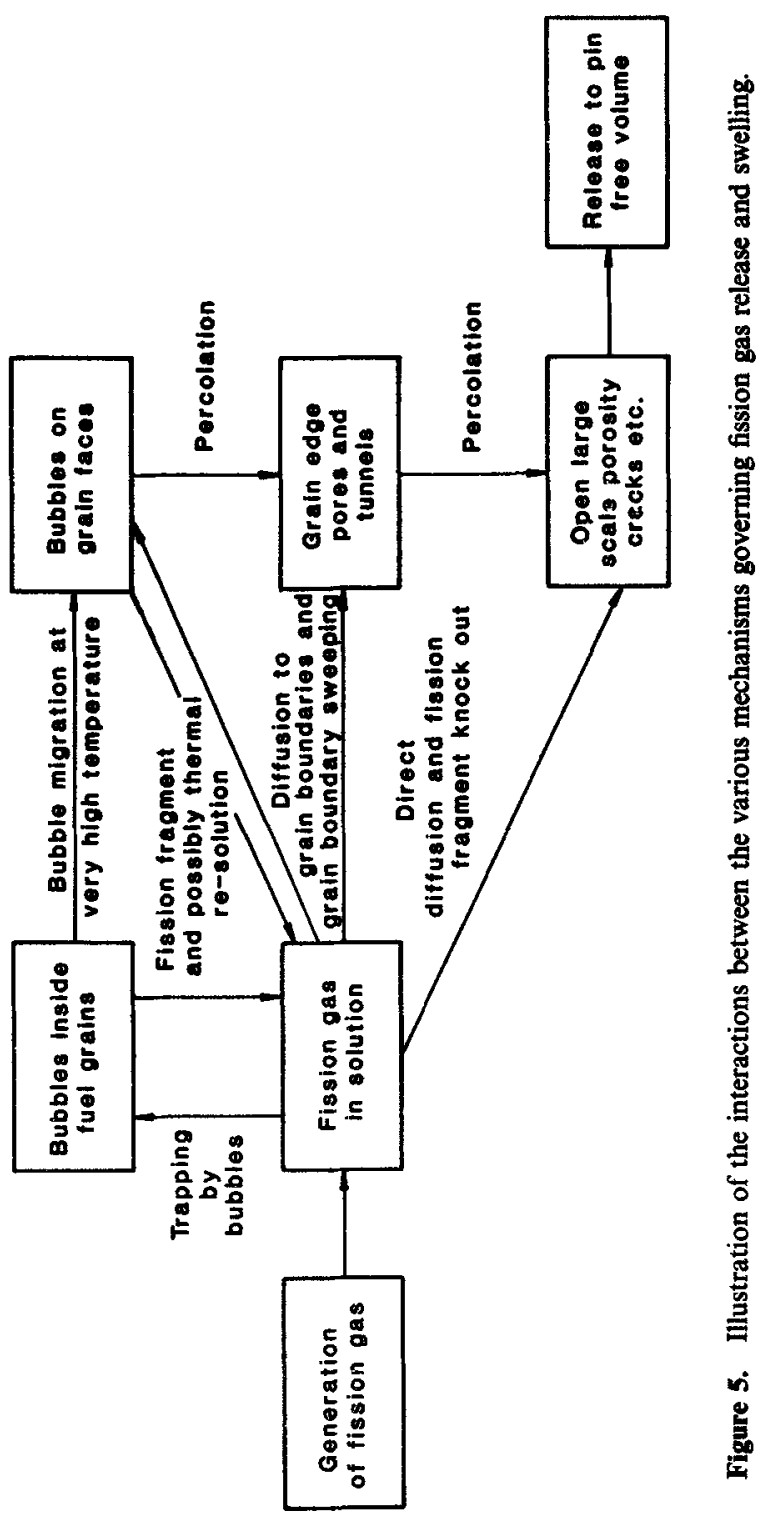


The most important new development is the introduction of the supercomputers (Duff 1984), which have recently increased the computing power available to fuel rod modellers by two orders of magnitude. This will enable calculations that were previously too expensive and long-running to be tackled. Two- and three-dimensional models of fuel behaviour will be introduced in cluster behaviour or even whole-core models. The basic $1 \frac{1}{2}$-dimensional fuel pin code will still have an important place in modelling, but it will be more frequently supplemented by two-dimensional finiteelement calculation to study stress concentrations and non axysymmetric effects. In particular it will be possible to couple the mechanics and thermal calculation directly for the whole fuel rod. This will remove the remaining sources of instability in the code and with proper vectorization of the calculation could be achieved with little cost penalty. It will then be possible to have improved treatments of coolant boiling, molten fuel motion and complex axial loadings coupled to the code. The TRAFIC code is being rewritten in this form at the moment for use on the Harwell CRAY computer.

\section{References}

Berna G A et al 1980 FRAPCON-2. A computer code for the calculation of steady-state thermo-mechanical behaviour of oxide fuel rods NUREG/CR-1845

Brearley I R et al 1984 Eur. Appl. Rept., Nucl. Sci. Technol. 51159

Bullough $\mathbf{R} 1984$ in Proc. Conf. on dislocations and properties and real materials, London (Royal Society, Metals Society)

Bullough R et al $1981 \mathrm{~J}$. Nucl. Mater. 103, 1041263

Cacciabue P C et al 1982 Comparative analysis of a hypothetical $0.15 /$ sec transient overpower accident in an irradiated LMFBR core using different computer models. Commission of the European Communities Report EUR 8018EN

Chatre A G 1983 Trans. 7th Int. Conf. on Structural Mechanics in Reactor Technology, Chicago, paper C3/6

Duff I S 1984 in Proc. Conf. on Vector and Parallel Processors in Computation and Science II, Oxford, UK

Dutt D S and Baker R B 1975 SIEX, A correlation code for the prediction of LMFBR fuel performance, Hanford Report HEDL-TME 74-55

Freidrich CM 1965 CYGRO Stress analysis of the growth of concentric cylinders, Bettis Atomic Power Labs. Report WAPD-TM-514

Gittus J H et al 1970 Nucl. Appl. Technol. 940

Gittus J H 1972 Nucl. Eng. Des. 1869

Gittus J H 1982 International experience and status of fuel element performance and modelling for water reactors, Springfields Nuclear Power Development Labs. Report ND-R 604(S)

Gittus J H 1984 Proc. Specialists Meeting on Water Reactor Fuel Element Performance Computer Modelling, Bowness-on-Windermere, UK, IAEA report IWGFPT/19, p. 15

Harriague $S$ et al 1980 Nucl. Eng. Des. 5683

Hermann L R 1965 A.I.A.A. July 3, 1896

Hughes $\mathrm{H}$ et al 1982 Fuel rod behaviour during transients Part 1. Description of codes, Springfields Report ND-R-702(S)

Ichikawa M et al 1980 Proc. Specialists Meeting on Fuel Element Performance Computer Modelling, Blackpool, UK, IAEA Report IWGFPT 7 p. 216

Jankus V Z and Weeks R W 1970 LIFE 1 - A Fortran IV computer code for the prediction of fast reactor fuel element behaviour. Argonne Report ANL 7736

Matthews J R 1972 Adv. Nucl. Sci. Technol. 667

Matthews J R 1979 Trans, 5th Int. Conf. on Structural Mechanics in Reactor Technology, Berlin, paper D7/1

Matthews J R 1983 J. Nucl. Eng. Des. (to be published)

Matthews J R 1984 The basis of the TRAFIC fuel performance code, Harwell Report AERE-R. 10818 (rev.)

Matthews J R et al 1985 The application of the TRAFIC fuel performance code to steady and transient conditions, BNEW Conf. on Nuclear Fuel performance, Stratford-upon-Avon, UK

Matthews J R and Wood M H 1984 Eur. Appl. Res. Rept., Nucl. Sci. Technol. 51685 
Melis J C et al 1982 PHSURA-A code for the interpretation of safety experiments, Int. Topical Meeting on LMFBR Safety and Related Design and Operational Aspects, Lyon, France p. II-45

Miller A K and Sherby O D 1977 Development of the Materials Code MATMOD (constitutive equations for Zircaloy) EPR NP 567

Nakatsuka M 1981 Nucl. Eng. Des. 65197

Nimo H and Ogawa S 1980 Proc. Specialists Meeting on fuel element performance computer modelling, Blackpool, UK, IAEA Report IWGFPT/7 paper 29

Preusser T and Lassmann K 1983 Trans. 7th Int. Conf. on Structural Mechanics in Reactor Technology, Chicago, paper $\mathrm{C} 4 / 3$

Rest J.1979 in Proc. 2nd Int. Seminar on Mathematical/Mechanical Modelling of Reactor Fuel Elements (Berlin: Bundersanstalt fur Materialprufung)

Sah D N and Venkatesh D 1984 Proc. Specialists Meeting on Water Reactor Fuel Element Performance Computer Modelling. Bowness-on-Windermere, UK, IAEA Report IWGFPT/19 p. 237

Schmidt C G and Miller A K 1981 Res. Mechanica 3 109, 175

Seifken L J 1983 Trans. 7th Int. Conf. on Structural Mechanics in Reactor Technology, Chicago paper C3/1.

Sontheimer F et al $1984 \mathrm{~J}$. Nucl. Mater. 12433

Stephen I D et al 1977 The LIFE code system for analysis of oxide fuel pin thermal/structural behaviour. Trans. Advanced LMFBR Fuels Topical Meeting, Tucson, Arizona

Stephen J D et al 1979 Proc. Specialists Meeting on Theoretical Modelling of LMFBR Fuel Pin Behaviour, Fontenay-aux-Roses, France, IAEA Report IWGFR/31, p. 139

Tromans N J and Matthews J R 1984 (unpublished work)

Van Vliet J and Billaux M 1984 Proc. Specialists Meeting on Water Reactor Fuel Element Performance Computer Modelling, Bowness-on-Windermere UK, IAEA Report IWGFPT/19 p. 151

Van Vliet J and de Meulemeester E 1980 Nucl. Eng. Des. 5671

Wider H U et al 1982 Status and validation of the SAS4A accident analysis code system. Int. Topical Meeting on LMFBR Safety and Related Design and Operational Aspects, Lyon, France p. II-13

Wilmore D and Matthews J R 1979 FRUMP-A physically based fuel model. Proc. Conf. on Fast Breeder Reactor Fuel, Monterey, California, p. 665

Wood M H et al 1984 Eur. Appl. Res. Rept., Nucl. Sci. Technol. 51515

Zimmermann H 1984 Eur. Appl. Res. Rept., Nucl. Sci. Technol. 51349 